isolated patients diagnosed with PVD. Data for patients who were missing important clinical identifiers (age, gender, race, mortality), did not receive IV thrombolysis, and had primary brain tumors (benign or malignant) were excluded. Data analyses assessed hospital mortality rate, length of stay (LOS), inpatient charges, and average age of admission.

Results Of the 24,692 encounters with AIS patients treated with thrombolysis and had no history of primary brain tumors, 4130 were diagnosed with PVD.

- Mean mortality rate was significantly increased (9.8\% PVD vs. $6.7 \%$ no PVD, $\mathrm{p}<0.0001)$.

- Mean LOS was significantly longer (7.35 days PVD vs. 6.26 days no PVD, $\mathrm{p}<0.0001$ ).

- Average total charges were significantly increased (\$101,115.04 PVD vs. \$84,908.15 no PVD, $\mathrm{p}<0.0001$ ).

- Average age at admission was significantly older (69.56 years PVD vs. 66.84 years no PVD, $\mathrm{p}<0.0001$ ).

Conclusion This study aims to inform physicians to better manage AIS patients receiving IV thrombolysis with PVD and no history of primary brain tumors. These patients experience higher mortality rate, longer LOS, increased total hospital charges, and older age at admission than those without PVD. These findings suggest that placing clinical focus on the coexisting condition of PVD before administering IV thrombolysis for AIS may be critical for improving short-term in-hospital outcomes. Future research should aim to investigate different thrombolytic agents to determine the most optimal choice for patients with peripheral vascular disease.

Disclosures K. Tong: None. A. Wang-Selfridge: None. P. Brauer: None.

\section{E-144 HIGH CLOT RED BLOOD CELL CONTENT IS ASSOCIATED WITH FIRST PASS SUCCESS IN MECHANICAL THROMBECTOMY}

1J Johnson*, ${ }^{1} \mathrm{~A}$ Srivatsan, ${ }^{2} \mathrm{~J}$ Chueh, ${ }^{3} \mathrm{M}$ Gounis, ${ }^{3} \mathrm{~A}$ Puri, ${ }^{1} \mathrm{~V}$ Srinivasan, ${ }^{4} \mathrm{~S}$ Chen, 1J Burkhardt, ${ }^{1} \mathrm{P}$ Kan. ${ }^{1}$ Neurosurgery, Baylor College of Medicine, Houston, TX; ${ }^{2}$ Radiology, University of Massachusetts, Worcester, MA; ${ }^{3}$ Radiology, University of Massachusetts Medical School, Worcester, MA; ${ }^{4}$ Radiology, Baylor College of Medicine, Houston, TX

10.1136/neurintsurg-2019-SNIS.219

Mechanical Thrombectomy has been associated with improved neurological outcome in large vessel occlusion (LVO) acute ischemic stroke (AIS) patients. To better understand the relationship of LVO clot composition with vessel recanalization, we analyzed individual clots from 94 retrieval passes in 43 AIS patients $(65.4 \pm 12.7$ years, 17 $(39.5 \%)$ female) who underwent intra-arterial mechanical thrombectomy procedures over a 2 -year period. Rates of successful vessel recanalization (TICI 2B - 3) for each trial and final result were analyzed. Clot composition was analyzed histologically, and the area occupied primarily by red blood cells (RBC), fibrin, or mixed was quantified. Fibrin $(44 \%)$ occupied the greatest area in retrieved clots, followed by mixed type (34\%) and RBCs (22\%). Clot composition was compared to recanalization success, first pass efficacy, patient comorbidities, devices or technique used, and carotid tortuosity score. In 43 patients, there were 94 passes (mean 2.14, range 1-6), 20 patients (47\%) had first pass successful revascularization, 41 (95\%) achieved successful revascularization, and 19 (48\%) had a good outcome at 3 months (mRS 0-2). In the univariate analysis, first pass efficacy was associated with RBC composition and Atrial Fibrillation but not with fibrin composition, mixed composition, type of first pass technique employed, distal emboli, good outcome, or carotid tortuosity score. In the multivariate analysis, first pass efficacy was significantly correlated with RBC composition $(p=.05)$ but not with atrial fibrillation $(\mathrm{p}=0.07)$ or other factors. Dense MCA sign on initial CT was correlated with mixed clot composition $(p=0.025)$. RBC clot composition is associated with first pass efficacy in patients undergoing mechanical thrombectomy for LVO.

Disclosures J. Johnson: None. A. Srivatsan: None. J. Chueh: None. M. Gounis: 1; C; NIH, NIH NINDS, Various Biomedical Device Industry Grants. A. Puri: None. V. Srinivasan: None. S. Chen: None. J. Burkhardt: None. P. Kan: 1; C; NIH, McDonald Foundation. 2; C; Stryker Neurovascular, Cerenovus, Medtronic.

\section{E-145 SASKATCHEWAN EXPERIENCE WITH MECHANICAL THROMBECTOMY UNDER GENERAL ANAESTHESIA}

A Persad*, S Ahmed, Z Tymchak, R Whelan, A Gardner, G Hunter, B Graham, L Peeling, M Kelly. University of Saskatchewan, Saskatoon, SK, Canada

10.1136/neurintsurg-2019-SNIS.220

Background While recent clinical trials have demonstrated immense efficacy of mechanical thrombectomy in the setting of acute stroke; there remains debate over the relative safety in performing this procedure under general anesthesia (GA). Recent RCT and meta-analyses have not provided a definitive answer to this question. With the reorganization of stroke systems of care, as a result of the Saskatchewan Acute Stroke Pathway, all patients presenting with LVO are assessed and endovascular thrombectomy is performed routinely under GA. We reviewed data from our pathway to add to the data regarding safety of thrombectomy under GA.

Methods Data was retrospectively reviewed on 154 consecutive LVO in 2016-2018 at the only Comprehensive stroke center in Saskatchewan. All patients undergoing MT were place under GA for the procedure. Pretreatment National Institutes of Health stroke scale (NIHSS), location of LVO and ASPECTS score were documented. Post-thrombectomy TICI scores, time to revascularization, and 90-day outcomes, both NIHSS and mRS, were recorded.

Results Of 154 LVO, 147 went on to have mechanical thrombectomy (MT). 65 were right anterior circulation, 70 were left anterior circulation and 11 were posterior circulation. Of 135 anterior circulation strokes, 69 (51.1\%), 58 $(42.9 \%)$ and $8(5.7 \%)$ had good, moderate and poor collateral circulation respectively, and the average pre-MT ASPECTS was 8.2. The average pre-MT NIHSS was 13.4. The average time from groin puncture to revascularization was $48.7 \mathrm{~min}$. A total of 133/147 (90.4\%) achieved thrombolysis in cerebral infarction (TICI) perfusion scale grade of $2 \mathrm{~b} / 3$ after recanalization. On follow-up, 70/147 and 90/ 147 had documented 90-day National Institutes of Health stroke scale (NIHSS) (average $=2.4$ ) and 90-day modified Rankin score $(\mathrm{mRS}$ ) (average $=2.2$ ). Overall mortality was 29/147 (19.7\%). 
Conclusions In a high volume Comprehensive Stroke Center, general anesthesia is safe to always use mechanical thrombectomy. Outcomes of mechanical thrombectomy for LVO in are in keeping with published results for both conscious sedation and general anesthesia. This adds to the body of evidence supporting GA as a excellent option for sedation for mechanical thrombectomy. It is particularly useful in more complex cases and leads to high revascularization rates.

Disclosures A. Persad: None. S. Ahmed: None. Z. Tymchak: None. R. Whelan: None. A. Gardner: None. G. Hunter: None. B. Graham: None. L. Peeling: 2; C; Medtronic. M. Kelly: 2; C; Medtronic.

\section{E-146 QUANTITATIVE EVALUATION OF FACTORS AFFECTING TUMOR SIZE REDUCTION ON WIDE ANGLE FUNDUS CAMERA FOLLOWING RETINOBLASTOMA CHEMOSURGERY}

${ }^{1} \mathrm{~S}$ Chen ${ }^{*},{ }^{2} \mathrm{P}$ Chévez-Barrios, ${ }^{3} \mathrm{M}$ Chintagumpala, ${ }^{3} \mathrm{~F}$ Lin, ${ }^{4} \mathrm{P}$ Kan, ${ }^{5} \mathrm{C}$ Area, ${ }^{6} \mathrm{C}$ Herzog, ${ }^{7} \mathrm{D}$ Gombos. ${ }^{1}$ Radiology and Neurosurgery, Baylor College of Medicine/Retinoblastoma Center of Houston, Houston, TX; ${ }^{2}$ Pathology, Retinoblastoma Center of Houston/Houston Methodist Hospital, Houston, TX; ${ }^{3}$ Pediatrics, Hematology-Oncology, Texas Children's Hospital/Retinoblastoma Center of Houston, Houston, TX; ${ }^{4}$ Neurosurgery, Baylor College of Medicine/Retinoblastoma Center of Houston, Houston, TX; ${ }^{5}$ Radiology, Baylor College of Medicine/Retinoblastoma Center of Houston, Houston, TX; ${ }^{6}$ Pediatrics, HematologyOncology, The University of Texas MD Anderson Cancer Center/Retinoblastoma Center of Houston, Houston, TX; ${ }^{7}$ Head and Neck Surgery, The University of Texas MD Anderson Cancer Center/Retinoblastomal Center of Houston, Houston, TX

\subsection{6/neurintsurg-2019-SNIS.221}

Introduction Intra-arterial delivery of chemotherapy (chemosurgery) via selective ophthalmic artery infusion has had an increasing role in the treatment of retinoblastoma. However reported success rates and enucleation rates have varied. The many differences in institutional protocols including choice of first line therapy, availability of patient follow-up and rationale for enucleation yields different results. We hypothesized that a quantitative assessment of tumor size reduction on wide angle funduscopic imaging would show differences in tumor response with regards to cycle of treatment, adjunct treatments, and prior IV chemotherapy.

Materials and methods Following IRB approval, a retrospective review of pediatric patients with retinoblastoma who had been referred for chemosurgery at our institution in the past 6 years was performed. Data collected included patient demographics, retinoblastoma classification, prior and concurrent treatments, chemotherapy agents, catheterization technique, fluoroscopy time, vascular anatomy, complications, and response on funduscopic examination. RetCam wide angle fundoscopic images were digitally transferred to DICOM image processing software for analysis or digitized from hard copy. Quantitative changes in tumor volume between images were measured.

Results From June 2013 to March 2019, a total of 114 chemosurgery cases were performed on 30

patients to treat 32 eyes. All patients received eye exams under anesthesia. 158 eye exams under anesthesia were reviewed including analysis of wide angle fundoscopic images when available.

After the first cycle of intra-arterial chemotherapy, response to treatment progressively decreased, with greatest change in tumor volume at time of first treatment. Decrease tumor size decrease per treatment was much greater when intra-arterial therapy was utilized as a first line therapy.

\begin{tabular}{llll} 
Abstract E-146 Table 1 & & & \\
\hline & Cycle & Cycle 2 & Cycle \\
& $\mathbf{1}$ & & $\mathbf{3}$ \\
\hline First Line & $58.9 \%$ & $35.54 \%$ & $2.05 \%$ \\
Prior IV & $4.12 \%$ & $3.97 \%$ & $2.88 \%$ \\
Chemotherapy & & & \\
\hline
\end{tabular}

Combination therapy appears to be more efficacious than IA alone for cycles 3 and greater.

\begin{tabular}{ll}
\hline IA Alone & $1.58 \%$ \\
\hline Concurrent Laser Hyperthermia & $2.89 \%$ \\
Concurrent Intravitreal & $9.30 \%$ \\
Chemotherapy & \\
\hline
\end{tabular}

The catheters were placed in two locations, either the middle meningeal artery or the origin of the ophthalmic artery. A slightly greater average decrease in tumor size per cycle was seen with middle meningeal infusion $(17.63 \%)$ versus ophthalmic artery infusion (12.38\%).

Conclusion Quantitative assessment of wide angle fundoscopic imaging for tumor size helps evaluate Chemosurgery efficacy retrospectively. Utilizing this measurement allows us to more accurately assess treatment effects, which appear to be greater in IV chemotherapy naïve patients.

Disclosures S. Chen: None. P. Chévez-Barrios: None. M. Chintagumpala: None. F. Lin: None. P. Kan: 1; C; Stryker Neurovascular, MacDonald Research Fund. 2; C; Stryker Neurovascular, Medtronic. C. Area: None. C. Herzog: None. D. Gombos: 1; C; Houseman/Wilkin Opthalmologicl Foundation. 2; C; Abbvie, Aura, 3T Ophthalmics. 6; C; Children's Oncology Group.

\section{E-147 A RETROSPECTIVE REVIEW OF THE IMPACT OF ASPIRIN USE IN SUBARACHNOID HEMORRHAGE}

${ }^{1}$ V Patel ${ }^{*},{ }^{1} \mathrm{~F}$ Al Mufti, ${ }^{2} \mathrm{R}$ Gupta. 'Neurosurgery, Westchester Medical Center, Valhalla, NY; ${ }^{2}$ Neurosurgery, Rutgers New Jersey Medical School, Newark, NJ

\subsection{6/neurintsurg-2019-SNIS.222}

Objective There is limited evidence on the use of aspirin (ASA) to reduce the risk and morbidity of cerebral aneurysmal rupture. This analysis retrospectively assessed aspirin use in patients presenting to our institution with aneurysm rupture.

Methods We evaluated 7 years of retrospective data from our neurovascular center and an existing database of patients with aneurysmal rupture. Initial presentation, hospital course and discharge outcomes were evaluated and compared based on whether or not patients were on ASA at the time of aneurysm rupture.

Results A total of 18 cases with patients on ASA and 168 control patients were identified. ASA use did not appear to lead to differences in initial presentation, including Hunt-Hess score and Fisher grade. In addition, ASA use was not associated with increased rates of delayed cerebral ischemia (DCI) or worsened modified Rankin score (mRs) at 90 days. Our analysis showed that increased Hunt Hess score and the 